\title{
Highly extinguished host galaxy of the dark GRB 020819
}

\author{
A. Küpcü Yoldaş ${ }^{1}$, J. Greiner ${ }^{2}$, S. Klose ${ }^{3}$, T. Krühler ${ }^{2,4}$, and S. Savaglio ${ }^{2}$ \\ ${ }^{1}$ European Southern Observatory, Karl-Schwarzschild-Str. 2, 85748 Garching, Germany \\ e-mail: ayoldas@eso.org \\ 2 Max-Planck-Institut für extraterrestrische Physik, Giessenbachstrasse 1, 85748 Garching, Germany \\ 3 Thüringer Landessternwarte Tautenburg, Sternwarte 5, 07778 Tautenburg, Germany \\ ${ }^{4}$ Universe Cluster, Technische Universität München, Boltzmannstrasse 2, 85748 Garching, Germany
}

Received 27 April 2010 / Accepted 6 May 2010

ABSTRACT

\begin{abstract}
We analyse the properties of the host galaxy of the optically dark gamma-ray burst (GRB) $020819(z=0.41)$ and discuss the possible implications in the context of "dark" GRBs. We present $g^{\prime} r^{\prime} i^{\prime} z^{\prime} J H K$ photometry of the host galaxy and fit the broad spectral energy distribution including the public Spitzer IRAC data using stellar population models. The broad spectral energy distribution (SED) indicates a high extinction, $A_{\mathrm{V}} \sim 1.8-2.6 \mathrm{mag}$, for this relatively massive galaxy. This is the highest global extinction for a GRB host galaxy with a robust spectroscopic redshift. The properties of the host galaxy are indicative of dusty, intense star-formation, which differ from those of the current sample of GRB hosts. This implies that the dust extinction is one of the main reasons for the darkness of low-redshift bursts and that the long GRB host population is far more diverse than previously anticipated.
\end{abstract}

Key words. gamma-ray burst: general - galaxies: ISM - galaxies: individual: GRB 020819 - galaxies: photometry

\section{Introduction}

The afterglows of gamma-ray bursts (GRBs) were predicted by the canonical fireball theory (e.g., Mészáros \& Rees 1997) and have since been detected for more than a decade (van Paradijs et al. 1997). The optical-to-X-ray flux ratio of a GRB afterglow is determined by the fireball theory (e.g., Sari et al. 1998), where the optical-to-X-ray spectral index of a canonical GRB afterglow is expected to be $\beta_{\mathrm{OX}} \geq 0.5$ (Jakobsson et al. 2004). However, for a significant proportion (25\%-50\%) of all well-localised GRBs, no optical/near-infrared afterglow is detected and/or the optical afterglow emission is lower than that expected from the X-ray afterglow emission $\left(\beta_{\mathrm{OX}}<0.5\right)$ (e.g., Fynbo et al. 2009; Cenko et al. 2009; de Pasquale et al. 2006). These bursts are called "dark" GRBs. The nature of the dark bursts is still to be understood, although several ideas have been proposed to explain why some bursts are dark in the optical bands: (i) shifting of the rest-frame optical afterglow emission and the Lyman-limit towards longer wavelengths for high redshift bursts; (ii) intrinsic dimness of the afterglow (for those with no optical emission and unknown $\beta_{\mathrm{OX}}$ ); or (iii) high extinction in either the host galaxy or the circumburst environment and along the line of sight (e.g., Fynbo et al. 2001; Lazzati et al. 2002; de Pasquale et al. 2003; Fox et al. 2003).

GRB $020819^{1}$ was detected by the High Energy Transient Explorer (HETE) satellite and identified as a moderately bright long-soft burst with $T_{90} \sim 20 \mathrm{~s}$ and a peak brightness of $\sim 5 \mathrm{crab}$ (Vanderspek et al. 2002). Follow-up ground-based observations led to a $K^{\prime}$-band limit of 19.5 mag and $R$-band limit of 22.15 for

\footnotetext{
1 This burst is in fact GRB 020819B (see Jochen Greiner's web site http://www .mpe.mpg.de/ jcg/grb020819.html). However it is referred to as GRB 020819 in all related GCNs and published papers, and we therefore also refer to it as GRB 020819 to avoid further confusion.
}

the afterglow emission $\sim 9 \mathrm{~h}$ after the burst (Klose et al. 2003; Levan et al. 2002). Therefore, GRB 020819 was classified as a dark burst.

Frail \& Berger (2002) later discovered a declining radio source consistent with radio afterglow emission located in a faint blob $3^{\prime \prime}$ away from the centre of a spiral galaxy (Jakobsson et al. 2005). This galaxy, at $z=0.41$, is identified as the host galaxy of GRB 020819 with a chance coincidence probability of $0.8 \%$ (Jakobsson et al. 2005). The host has a visible extent of $\sim 6^{\prime \prime}-7^{\prime \prime}$ (32.5-38 kpc) in the $R$-band (see Fig. 1$)$.

Here we present broad-band photometric observations of the host galaxy of GRB 020819 (Sect. 2) and the analysis of its spectral energy distribution (Sect. 3). In Sect. 4, we discuss the results and their implications for the dark GRB framework.

\section{Observations}

The host galaxy of GRB 020819 was observed with GROND (Greiner et al. 2008) in $g^{\prime} r^{\prime} i^{\prime} z^{\prime} J H K$ on 29 October 2007 for $\sim 1 \mathrm{~h}$ at an average airmass of 1.23 . The data reduction and aperture photometry of the host was performed using the data reduction and photometry tools of the GROND pipeline (see e.g. Küpcü Yoldaş et al. 2008) based on IRAF/PyRAF. Astrometry and photometric calibration was performed using the SDSS catalogue (Abazajian et al. 2009) for the optical bands, and the 2MASS catalogue (Skrutskie et al. 2006) for infrared bands.

The brightness of the host galaxy was derived using aperture photometry with a radius of $\sim 4.5^{\prime \prime}(3 \times \mathrm{PSF})$ in all bands. The results of the photometry are shown in Table 1. All magnitudes are corrected for a foreground Galactic extinction of $E(B-V)=0.069$ (Schlegel et al. 1998). We note that our $g^{\prime}$-band magnitude, $20.31 \pm 0.02$, is brighter than the $B$-band magnitude, $21.9 \pm 0.5$, of the host reported by Jakobsson et al. (2005). The difference is larger than that expected because of the differences 
Table 1. The photometry of the host galaxy of GRB $020819^{a}$.

\begin{tabular}{ccccccc}
\hline \hline$g^{\prime}$ & $r^{\prime}$ & $i^{\prime}$ & $z^{\prime}$ & $J$ & $H$ & $K$ \\
\hline $20.31 \pm 0.02$ & $19.43 \pm 0.06$ & $19.10 \pm 0.07$ & $18.73 \pm 0.01$ & $18.46 \pm 0.04$ & $18.23 \pm 0.04$ & $18.46 \pm 0.11$ \\
\hline
\end{tabular}

Notes. ${ }^{(a)}$ All magnitudes are in units of $A B$ mag and are corrected for foreground Galactic extinction.

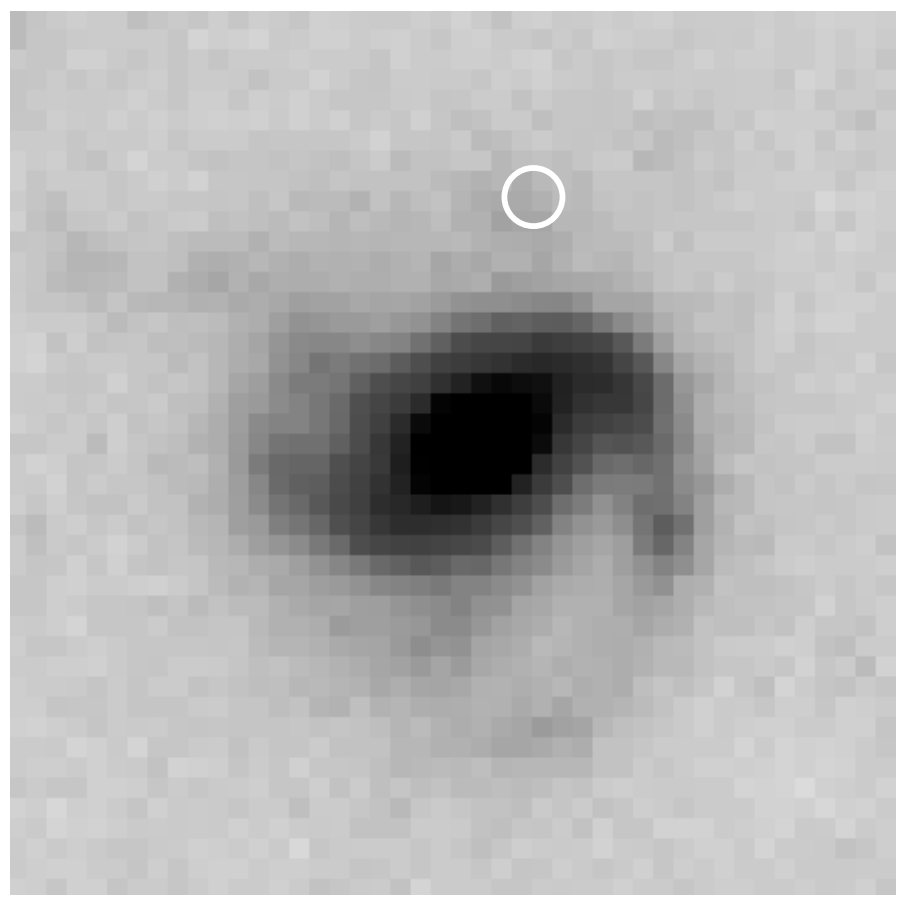

Fig. 1. VLT/FORS2 $R$-band image of the host galaxy of GRB 020819 taken on 15 Sep. 2002. It is clearly resolved as a barred spiral galaxy with bright knots of star-formation in its spiral arms and with its shape possibly resembling NGC 1300 . The approximate position of the radio transient is shown by the circle (following Jakobsson et al. 2005). The field of view is $11 \times 11 \operatorname{arcsec}^{2}$. North is up, east is left.

between the two filter curves. The reason for this difference is probably the inaccurate calibration of the Keck $B$-band data where Jakobsson et al. (2005) used a single faint star for calibration, which was the only available star in the field (Jakobsson, private communication).

\section{Spectral energy distribution}

The spectral energy distribution (SED) was analysed using HyperZ (Bolzonella et al. 2000). The redshift of the host was fixed to the spectroscopic value of $z=0.41$. We allowed the extinction value $A_{\mathrm{V}}$ to vary and fit the host extinction by using 4 different reddening laws: MW, LMC, SMC, and Calzetti (Calzetti et al. 2000). To fit the stellar populations, we used two different stellar population model libraries: the GISSEL library of Bruzual \& Charlot (1993) and the stellar population models of Maraston (2005). The initial mass function of all models follows the Salpeter law (Salpeter 1955).

The GISSEL library contains models with 8 different starformation histories: an instantaneous burst, a constant starforming system, and six exponentially decaying star-formation rate $(\mathrm{SFR})$ models where $S F R \propto \exp (-t / \tau), \tau$ being the characteristic lifetime. There are two sets of stellar population models in the GISSEL library: i) those with metallicities fixed to the solar value; and ii) those for which the metallicity evolves, where the evolution in metallicity of the stellar population is explicitly taken into account so that there is a natural link between age and mean metallicity (Bolzonella et al. 2000). The ages of the templates vary between zero and the age of the Universe (at $z=0.41$ in the case of this GRB) covered with unevenly distributed steps (Bolzonella et al. 2000).

From Maraston (2005) models, we used both single and composite stellar population models. We selected the models with metallicity $Z=0.04$ (twice the solar value) to maintain agreement with the metallicity measured based on the spectroscopic line ratio of $[\mathrm{NII}] \lambda 6584 /[\mathrm{OII}] \lambda 3727$, leading to $\log (\mathrm{O} / \mathrm{H})+12=9.0 \pm 0.1$ (Levesque et al. 2010). The single stellar population (SSP) models that we used have a red horizontal branch morphology, meaning that the entire horizontal branch lifetime is spent on the red side of RR Lyrae strip (Maraston 2005).

The host galaxy of GRB 020819 was also observed using IRAC on-board the Spitzer satellite. Svensson et al. (2010) measured the IRAC $3.6 \mu \mathrm{m}$ and $5.8 \mu \mathrm{m}$ brightnesses as $18.96 \pm$ $0.02 \mathrm{mag}$ (corresponding to $95.1 \pm 1.8 \mu \mathrm{Jy}$ ) and $19.27 \pm 0.22 \mathrm{mag}$, respectively. Castro Ceron et al. (2008) also reports the IRAC $3.6 \mu \mathrm{m}$ band brightness as $97 \pm 2 \mu \mathrm{Jy}$, in agreement with Svensson et al. (2010). We used these measurements with our GROND data to form a broad SED that covers the rest-frame UV to infrared wavelengths. In the following paragraphs, we present the SED fits of the host galaxy of GRB 020819, using GROND data combined with the IRAC brightnesses. The errors quoted in the following sections are at the $1 \sigma$ level and calculated by taking into account all the galaxy templates of a given stellar population library used in that fit.

\subsection{Fits to the GROND and IRAC data}

The best-fit models to the broad-range SED formed by GROND and IRAC data, are those with a MW-like extinction for both stellar library models (see Fig. 2). Using the models with evolving metallicity from the GISSEL library, the best fit galaxy model has an age $30_{-15}^{+60.5} \mathrm{Myr}$ and an extinction of $A_{\mathrm{V}}=2.21 \pm$ 0.39 mag using a MW reddening law and an exponentially decaying SFR with $\tau=1 \operatorname{Gyr}\left(\chi_{v}^{2}=2.73\right)$. The stellar mass of the best-fit model is $\sim 10^{10.4}$ solar masses, in agreement with Savaglio et al. (2009).

Using Maraston SSP and CSP models with a fixed metallicity of $Z=0.04$, the best-fit is obtained with a CSP model that has an exponentially decaying SFR with $\tau=1 \mathrm{Gyr}$, age $6.0 \pm 0.5 \mathrm{Myr}$ and $A_{\mathrm{V}}=2.47_{-0.13}^{+0.26}$ using a MW reddening law. The models with longer SFR e-folding times, $\tau$, provided similarly good fits with the same age and extinction values as best-fit. All other reddening laws provided a significantly $(>1 \sigma)$ poorer fit. SSP models also fit similarly well but only when using a Calzetti reddening law with a best-fit model of $A_{\mathrm{V}}=3.12$ and age $=3 \mathrm{Myr}$.

We note that when using a MW reddening law, even the best fit does not provide a good fit to the $H$-band (see Fig. 2). 


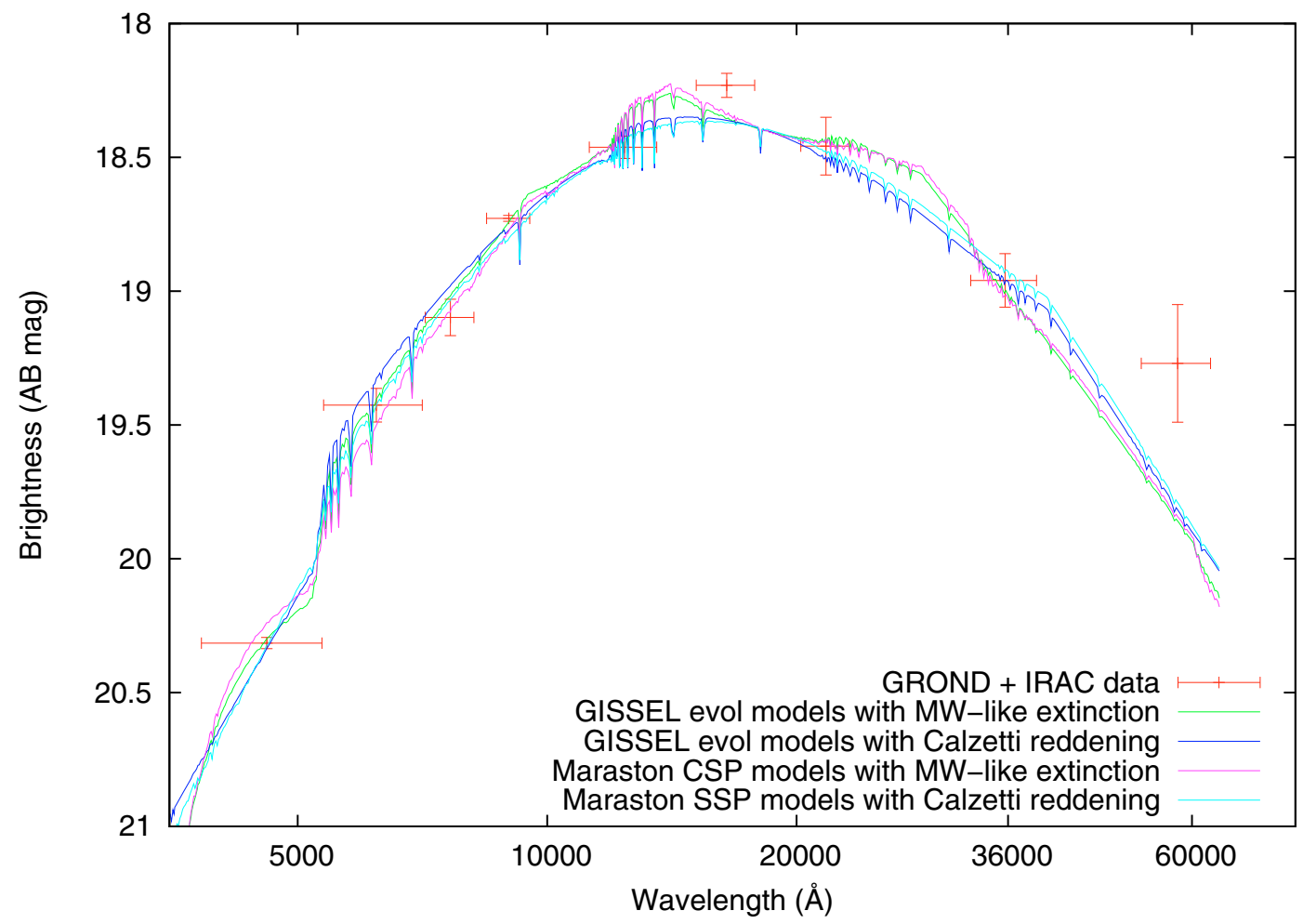

Fig. 2. The fits to the SED of the host of GRB 020819 using GROND and IRAC data. Note that we repeated the fits after increasing the IRAC $3.6 \mu \mathrm{m}$ band brightness error from $0.02 \mathrm{mag}$ to $0.1 \mathrm{mag}$. Error bars for both cases are shown in the figure.

Similarly, the stellar population models extinguished with the Calzetti reddening law overestimate the $K$-band flux. The reason for the imperfect fits to the $H$-band flux is possibly emission lines such as $\mathrm{Pa} \beta$ and $\mathrm{He}$ I from the ionised interstellar gas, which fall into the $H$-band at the redshift of this galaxy.

\section{Results and discussion}

The SED fits show that the optical and near-infrared emission from the galaxy is dominated by a very young stellar population. The young age of the dominant stellar population prevents us from distinguishing between models with different SFR efolding times and hence does not allow us to determine the SFR history of the galaxy. The age of the dominant stellar population is $\sim 6 \mathrm{Myr}$, if the metallicity is fixed to $Z=0.04$, and slightly older, $30 \mathrm{Myr}$, if the metallicity evolves in proportion to the age of the stellar population. The MW-like extinction provides better fits to the data than the Calzetti reddening. The best-fit extinction value is in the range $A_{\mathrm{V}} \sim 1.8-2.6$ mag depending on the galaxy template library used.

In principle, there is a degeneracy between the dust extinction in a galaxy and the age and metallicity of the mean stellar population of a galaxy, since all three redden the colour of the galaxy. However, it is also known that metal-poor galaxies have less dust and lower global infrared emission (Calzetti et al. 2010; Cannon et al. 2005, 2006; Walter et al. 2007). This is supported by our finding that the dust extinction and the age values obtained by fitting the solar/super-solar metallicity models and the evolving metallicity models roughly agree with each other, indicating that either the metallicity is high or it does not have a significant effect on the determination of the dust extinction for this galaxy, or both. In other words, the galaxy is highly extinguished according to the fits by synthetic stellar population models, independent of the metallicity of the models.
The age and dust extinction values we obtained agree with the spectroscopic measurements of Levesque et al. (2010) who found an age of 7.8 \pm 0.9 Myr using the $\mathrm{H}_{\beta}$ emission line equivalent width and $E(B-V)=0.64$ using the $\mathrm{H}_{\alpha} / \mathrm{H}_{\beta}$ line ratio. For a MW-like total-to-selective extinction ratio $R_{V}=3.1$, $E(B-V)=0.64$ corresponds to $A_{\mathrm{V}}=1.98 \mathrm{mag}$, which is in agreement with the $A_{\mathrm{V}}$ values from our SED fits. Levesque et al. (2010) also measured the metallicity of the host galaxy based on the spectroscopic line ratio of [NII] $\lambda 6584 /[\mathrm{OII}] \lambda 3727$ and found a super-solar metallicity of $\log (\mathrm{O} / \mathrm{H})+12=9.0 \pm 0.1$.

The age (and extinction) value obtained from either our SED fits or the spectrum, by Levesque et al. (2010), do not agree with that of Savaglio et al. (2009). The reason for this disagreement is probably that they fit the SED of this host using only the $B-, R$-, and $K$-band magnitudes available in the literature, where the $B$-band magnitude has a large uncertainty (see Sect. 2). Furthermore, their fits are restricted to the $A_{\mathrm{V}}$ range of 0.0-2.0 mag and metallicities no more than the solar value, which do not agree with the best-fit model $A_{\mathrm{V}}$ or metallicity of this host. Hence, our analysis illustrates the importance of accurate photometry, a broad SED coverage, and correctly selected parameter ranges.

On the other hand, our best-fit stellar mass of $\sim 10^{10.4} M_{\odot}$ agrees with $\sim 10^{10.50 \pm 0.14} M_{\odot}$ of Savaglio et al. (2009), indicating that the stellar mass calculations based on the optical/nearinfrared SED are much less affected by the choice of parameter ranges. A reason for the robustness of the mass estimation is that the SED covers the Balmer break and the rest-frame nearinfrared emission of the galaxy.

The calculation of the dust extinction based on the afterglow emission limits of GRB 020819 in the $R$ - and $K^{\prime}$-band by Klose et al. (2003) show that if the afterglow is similar to that of GRB 011121 and at the same redshift $(z=0.36)$, then an extinction of $A_{\mathrm{V}} \sim 4 \mathrm{mag}$ is required to $\operatorname{dim}$ the $R$-band afterglow and 
$A_{\mathrm{V}} \sim 20$ mag to dim the $K^{\prime}$-band afterglow below the limits. We performed a fit to the afterglow upper limits of the $R$ - and $K^{\prime}$ band at $z=0.41$ using a host galaxy extinction of $A_{\mathrm{V}}=2.2 \mathrm{mag}$ (following a MW-like extinction law) and found that the afterglow should have a spectral decay index $\beta>0.82$. This value is very close to the canonical value of 0.8 , showing that the afterglow of this dark GRB is probably quite normal i.e., not significantly different from other long GRB afterglows. Therefore, the "darkness" of this GRB is probably not related to the properties of its afterglow but the dust in the host galaxy. The high $A_{\mathrm{V}}$ value is consistent with the darkness of the GRB afterglow, although the $A_{\mathrm{V}}$ that we deduced here is not only for the GRB region but the whole galaxy.

There are several bursts with high extinction in the circumburst region or along the line of sight, derived using the afterglow emission (e.g. Djorgovski et al. 2001; Rol et al. 2007; Jaunsen et al. 2008; Tanvir et al. 2008; Kann et al. 2006, 2009; Hashimoto et al. 2010). However, GRB 020819 is the first case with known redshift and a well-constrained high extinction value for the entire galaxy. A similar case is that of GRB 090417B $(z=0.345)$ for which Holland et al. (2010) measure $A_{\mathrm{V}} \sim 3.5 \mathrm{mag}$ based on the UV and optical SED of this dark GRB host. The high hostgalaxy extinctions of these low-redshift dark GRBs also show that while the dust extinction is one of the main reasons for the darkness of low-redshift bursts, at high-redshift $(z>1)$ the dust extinction required to dim the afterglow is not that high.

The case of GRB 020819 is an exception in the context of both the host galaxies of long GRBs with bright optical afterglows, and the dark GRB hosts. Host galaxy studies of dark GRBs indicate that the majority of the dark GRB hosts are similar to normal long GRB hosts, which do not have very high extinction or very high redshifts (Perley et al. 2009). However, the host extinction of GRB 020819 is much higher than the average extinction, $A_{\mathrm{V}} \leq 0.5$, of a sample of hosts galaxies of long GRBs with detected optical afterglows, obtained either using spectroscopy or SED fitting (e.g., Han et al. 2010; Savaglio et al. 2009). Furthermore, the spectroscopy of the host indicates very high star-formation-rate (SFR) of $23.6 M_{\odot} / \mathrm{yr}$ (Levesque et al. 2010) corresponding to a specific SFR (SFR/stellar mass) of 0.94 per $\mathrm{Gyr}^{-1}$ using our best-fit stellar mass $10^{10.4} M_{\odot}$. This specific SFR is higher than the median specific $S F R \sim 0.8 \mathrm{Gyr}^{-1}$ of long GRB host galaxies (Savaglio et al. 2009). These properties mark the host galaxy of GRB 020819 as a galaxy with dusty, intense star-formation, distinguished from the currently known GRB host population.

The host galaxy of another dark GRB $051022(z=0.807)$ shows similarly exceptional properties in terms of mass $\left(\sim 10^{10.4} M_{\odot}\right)$, SFR $\left(\sim 36 M_{\odot} / \mathrm{yr}\right)$ and metallicity $(\log (\mathrm{O} / \mathrm{H})+$ 12 = 8.77) (Savaglio et al. 2009; Graham et al. 2009). We note however that there is a large uncertainty in the metallicity calculation for this host depending on whether the upper branch solution calibrated with metal-rich galaxies or a lower branch solution calibrated with metal-poor galaxies is used (see e.g., Kobulnicky et al. 1999; Kewley \& Dopita 2002). The extinction of the host galaxy of GRB $051022\left(A_{\mathrm{V}}=1.55\right.$, Levesque et al. 2010) is also relatively high compared to the average long-GRB host population. Similarly, there are a few dark GRBs that have both high extinction and near-solar metallicities derived using afterglow observations (e.g., Watson et al. 2006; Krühler et al. 2008; Elíasdóttir et al. 2009; Prochaska et al. 2009), indicating that hosts such as that of GRB 020819 may be more common than previously anticipated.

These findings and the clear example of the host galaxy of GRB 020819, show that the hosts of GRBs may be far more diverse, in terms of extinction, mass and metallicity, than previously found.

Acknowledgements. We thank Paulo Afonso for his help on carrying out the observations, and the referee for useful comments and suggestions. T.K. acknowledges support by the DFG cluster of excellence "Origin and Structure of the Universe". Part of the funding for GROND (both hardware as well as personnel) was generously granted from the Leibniz-Prize to Prof. G. Hasinger (DFG grant HA 1850/28-1).

\section{References}

Abazajian, K. N., Adelman-McCarthy, J. K., Agüeros, M. A., et al. 2009, ApJS, 182,543

Bolzonella, M., Miralles, J.-M., \& Pello, R. 2000, A\&A, 363, 476

Bruzual, G., \& Charlot, S. 1993, ApJ, 405, 538

Calzetti, D., Armus, L., Bohlin, R. C., et al. 2000, ApJ, 533, 682

Calzetti, D., Wu, S.-Y., Hong, S., et al. 2010, ApJ, 714, 1256

Cannon, J. M., Walter, F., Bendo, G. J., et al. 2005, ApJ, 630, L37

Cannon, J. M., Smith, J. T. D., Walter, F., et al. 2006, ApJ, 647, 293

Castro Cerón, J. M., Michaĺowski, M. J., Hjorth, J., et al. 2008, ApJ, submitted, [arXiv:0803.2235]

Cenko, S. B., Kelemen, J., Harrison, F. A., et al. 2009, ApJ, 693, 1484 de Pasquale, M., Piro, L., Perna, R., et al. 2003, ApJ, 592, 1018 de Pasquale, M., Piro, L., Gendre, B, et al. 2006, A\&A, 455, 813 Djorgovski, S. G., Frail, D. A., Kulkarni, S. R., et al. 2001, ApJ, 562, 654 Elíasdóttir, Á., Fynbo, J. P. U., Hjorth, J., et al. 2009, ApJ, 697, 1725 Frail, D. A., \& Berger, E. 2002, GCN Cric., 1842

Fox, D. W., Price, P. A., Soderberg, A. M., et al. 2003, ApJ, 586, L5 Fynbo, J. U., Jensen, B. L., Gorosabel J., et al. 2001, A\&A, 369, 373 Fynbo, J. P. U., Jakobsson, P., Prochaska, J. X., et al. 2009, ApJS, 185, 526 Graham, J. F., Fruchter, A. S., Kewley, L. J., et al. 2009, AIPC, 1133, 269 Greiner, J., Bornemann, W., Clemens, C., et al. 2008, PASP, 120, 405 Han, X. H., Hammer, F., Liang, Y. C., et al. 2010, A\&A, 514, A24 Hashimoto, T., Ohta, K., Aoki, K., et al. 2010, ApJ, submitted, [arXiv: $1003.3717 \mathrm{v} 1]$

Holland, S., Sbarufatti, B., Cummings, J. R., et al. 2010, AAS, 215, 303.01 Jakobsson, P., Hjorth, J., Fynbo, J. P. U., et al. 2004, ApJ, 617, L21 Jakobsson, P., Frail, D. A., Fox, D. B., et al. 2005, ApJ, 629, 45 Jaunsen, A. O., Rol, E., Watson, D. J., et al. 2008, ApJ, 681, 453 Kann, D. A., Klose, S., \& Zeh, A. 2006, ApJ, 641, 993

Kann, D. A., Klose, S., Zhang, B., et al. 2009, ApJ, submitted, [arXiv:0712.2186v2]

Kewley, L. J., \& Dopita, M. A. 2002, ApJS, 142, 35

Klose, S., Henden, A. A., Greiner, J., et al. 2003, ApJ, 592, 1025

Krühler, T., Küpcü Yoldaş, A., Greiner, J., et al. 2008, ApJ, 685, 376

Kobulnicky, H. A., Kennicutt, R. C., \& Pizagno, J. L. 1999, ApJ, 514, 544

Küpcü Yoldaş, A., Krühler, T., Greiner, J., et al. 2008, AIPC, 1000, 227

Lazzati, D., Covino, S., \& Ghisellini, G. 2002, MNRAS, 330, 583

Levan, A., Burud, I., Fruchter, A., et al. 2002, GCN Circ., 1517

Levesque, E., Kewley, L., Graham, J., \& Fruchter, A. S. 2010, ApJ, 712, L26

Maraston, C. 2005, MNRAS, 362, 799

Mészáros, P., \& Rees, M. 1997, ApJ, 476, 232

Perley, D., Cenko, S. B., Bloom, J. S., et al. 2009, AJ, 138, 1690

Prochaska, J. X., Sheffer, Y., Perley, D. A., et al. 2009, ApJ, 691, L27

Rol, E., van der Horst, A., Wieserma, K., et al. 2007, ApJ, 669, 1098

Salpeter, E. E. 1955, ApJ, 121, 161

Sari, R., Piran, T., \& Narayan, R. 1998, ApJ, 497, L17

Savaglio, S., Glazebrook, K., \& Le Borgne D. 2009, ApJ, 691, 182

Schlegel, D., Finkbeiner, D., \& Davis, M. 1998, ApJ, 500, 525

Skrutskie, M. F., Cutri, R. M., Stiening, R., et al. 2006, AJ, 131, 1163

Svensson, K. M., Levan, A. J., Tanvir, N. R., Fruchter, A. S., \& Strolger, L.-G. 2010, MNRAS, 405, 57

Tanvir, N. R., Levan, A. J., Rol, E., et al. 2008, MNRAS, 388, 1743

Vanderspek, R., Villasenor, J., Jernigan, J. G., et al. 2002, GCN Circ., 1508

van Paradijs, J., Groot, P. J., Galama, T., et al. 1997, Nature, 386, 686

Walter, F., Cannon, J. M., Roussel, H., et al. 2007, ApJ, 661, 102

Watson, D., Fynbo, J. P. U., Ledoux, C., et al. 2006, ApJ, 652, 1011 